\title{
Efecto de una $\alpha$ amilasa (ec 3.2.1.1) en el patrón de consumo y eficiencia productiva de corderos alimentados con una dieta alta en concentrado
}

\author{
Effect of $\alpha$ amylase (ec 3.2.1.1) on intake pattern and performance \\ of lambs fed a high concentrate diet \\ FX Plata ${ }^{a}$, PA Hernández ${ }^{\text {b }}$ GD Mendoza ${ }^{a}$, JA Martínez ${ }^{\text {a }}$ \\ aDepartamento de Producción Agrícola y Animal, Universidad Autónoma Metropolitana, DF, México. \\ ${ }^{\mathrm{b}}$ Centro Universitario Amecameca, Universidad Autónoma del Estado de México, DF, México.
}

\begin{abstract}
SUMMARY
The objective of this experiment was to evaluate the addition of an $\alpha$ amylase to the diet on the performance (average daily gain (ADG), dry matter intake (DMI) and feed conversion (FC), apparent dry matter digestibility (DMD), intake pattern and net income of lambs fed with a high grain diets. The experiment was conducted using 20 male lambs (BW $24.36 \pm 2.3 \mathrm{~kg}$ ), allocated in a completely randomized design: Control $(\mathrm{C})=70 \%$ commercial concentrate and $30 \%$ alfalfa hay, Enzyme (ENZ) $=\mathrm{C}+\alpha$ amylase. Lambs were housed in individual cages where performance and intake pattern were determined for 56 days; DMD was determined at the end of the experiment. Furthermore, the number of days and animals with reduced feed intake was estimated and economic analysis of the entire experiment was made. The addition of enzyme to the diet tended to reduce the DMI $(\mathrm{C}, 1.21 \mathrm{~kg} / \mathrm{d}$ and ENZ $1.13 \mathrm{~kg} / \mathrm{d} ; \mathrm{P}<0.07)$ during the finishing period, the ADG and the FC were similar between treatments $(\mathrm{P}=0.32, \mathrm{P}=0.095)$. The number of animals with a reduced feed intake more than $35 \%$ was higher in ENZ than in the control group (Fisher: $p=1, R^{2}=1$ ). Feed costs with ENZ were higher than those of $\mathrm{C}$ group $(\mathrm{P}<0.05)$. The increase in feed costs reduced net economic benefit with the $\alpha$ amylase.
\end{abstract}

Key words: amylase, profit, feed intake, lambs.

\section{RESUMEN}

El objetivo de esta investigación fue evaluar la adición a la dieta de una $\alpha$ amilasa en el desempeño (ganancia de peso (GDP), consumo de alimento (CMS) y conversión alimentaria (CA), la digestibilidad aparente de la materia seca (DMS), el patrón de consumo de alimento y la utilidad neta de corderos alimentados con una dieta alta en concentrado. El experimento se realizó con 20 borregos machos (PV 24,36 $\pm 2,3 \mathrm{~kg}$ ), asignados en un diseño completamente al azar a los siguientes tratamientos: control $(C)=70 \%$ de concentrado comercial y $30 \%$ de heno de alfalfa, enzima $($ ENZ) $=$ $\mathrm{C}+\alpha$ amilasa. Los corderos fueron alojados en jaulas individuales donde el desempeño y el patrón de consumo de materia seca fueron determinados durante 56 días, al final del experimento se determinó la DMS. Además, se estimó el número de días y de animales que tuvieron un consumo reducido de alimento y se realizó el análisis económico del experimento. La adición de enzima a la dieta tendió a reducir el CMS (C, 1,21 kg/d y ENZ 1,13 kg/d; $\mathrm{P}<0,07)$, la GDP y CA fueron similares entre los tratamientos $(\mathrm{P}=0,32 ; \mathrm{P}=0,095)$. El número de animales con un consumo reducido en más del 35\% fue mayor con ENZ que en el grupo C (Fisher: $\mathrm{P}=1 ; \mathrm{R}^{2}=1$ ). Los costos de alimentación en el grupo ENZ fueron mayores a los del grupo $\mathrm{C}(\mathrm{P}<0,05)$. Este aumento en el costo de alimentación redujo la utilidad económica con la $\alpha$ amilasa.

Palabras clave: amilasa, consumo, corderos, utilidad económica.

\section{INTRODUCCIÓN}

El aumento de la competencia en la industria ganadera ha llevado a los productores a buscar nuevos productos con el objetivo de mejorar la eficiencia de la producción. Una tecnología prometedora es la inclusión de enzimas exógenas en las dietas de los animales (Tricarico y col 2008). Varios estudios que utilizaron combinaciones de enzimas fibrolíticas en bovinos alimentados con dietas basadas en forraje han reportado efectos benéficos, como el incremento en la digestibilidad aparente de la MS, el aumento de la producción de leche y mejora en

Aceptado: 20.08.2014.

* Calz. del Hueso 1100, Villa Quietud, Coyoacán, DF. México, CP 04970; ppfx2221@ correo.xoc.uam.mx la ganancia diaria de peso (Pinos y col 2005, GómezVázquez y col 2011).

La intensificación de los sistemas de producción animal se basa en el uso de granos o concentrados (Blanch y col 2009), por esta razón, aumentar la digestión ruminal del almidón es uno de los factores más importantes que determina el rendimiento de los rumiantes (Huntington 1997). Se han desarrollado varias técnicas de procesamiento de grano para aumentar la tasa de digestión de almidón (Owens y col 1997). Dentro de estas técnicas se incluye la adición de enzimas amilolíticas (Rojo y col 2005), lo que permite disminuir el nivel de grano en la dieta (Mota y col 2011).

El uso de enzimas amilolíticas en los alimentos ha mejorado la digestibilidad de la materia seca (MS) en los cerdos, aves y corderos alimentados con dietas altas en 
grano (Rode y col 2002, Colombatto y col 2003, Rojo y col 2005). Las enzimas amilolíticas pueden aumentar la tasa de la digestión del almidón en algunos granos de digestión lenta, como el sorgo (Crosby y col 2006) y por lo tanto mejorar el rendimiento; sin embargo, el incremento en la velocidad de fermentación o en la cantidad de carbohidratos fermentados rápidamente resulta en la acumulación ruminal de ácidos grasos volátiles (AGV) que reducen el $\mathrm{pH}$, por lo que pueden aumentar el riesgo de acidosis ruminal clínica o subclínica (Blanch y col 2009). Algunos autores han considerado que un animal sufre acidosis subclínica cuando su consumo se reduce más del 35\% y presenta acidosis clínica si el consumo es menor al $80 \%$ de su consumo promedio (Brown y col 2000). Otros investigadores analizaron el efecto de la $\alpha$ amilasa en la digestibilidad in vitro o in situ de la materia seca de la dieta de ganado bovino y encontraron una respuesta positiva (Buendía y col 2003). Sin embargo, este tipo de enzima no ha demostrado beneficios productivos cuando es incluida en las dietas de finalización de corderos o novillos (Mota y col 2011, DiLorenzo y col 2011), por lo que se necesita más información para su uso en este tipo de dietas. Por esta razón, el objetivo de este trabajo fue evaluar los efectos de una $\alpha$ amilasa comercial en la ganancia de peso, el patrón y cantidad de alimento consumido, la eficiencia alimentaria, la digestibilidad aparente de la materia seca, los días que se altera el consumo de alimento como indicadores de acidosis subclínica y la utilidad neta de borregos que se alimentan con una dieta alta en concentrado.

\section{MATERIAL Y MÉTODOS}

El experimento se realizó en la Universidad Autónoma de Chapingo; en la unidad experimental "El 13" (Estado de México). Se utilizaron veinte corderos cruza de Pelibuey $x$ Kathahdin con un peso inicial promedio de $24,3 \pm 2,3$ $\mathrm{kg}$. Los animales fueron alojados individualmente y asignados en un diseño completamente al azar a uno de los siguientes tratamientos: control $(\mathrm{C} ; \mathrm{n}=10)$ compuesto por: $70 \%$ de concentrado comercial (Engorda cordero plus ${ }^{\circledR}$; Productores Agropecuarios Tepexpan S.A. de C.V.) y $30 \%$ de heno de alfalfa; Enzima (ENZ; n $=10)$ como C + enzima. La solución de enzima $\left(\right.$ Hyrazime $^{\circledR}$ de ENMEX) fue diluida en agua en una relación (3:1) y fue rociada en el concentrado comercial a una dosis de 2,3 $\mathrm{g}$ de producto / $\mathrm{kg}$ de MS.

\section{COMPOSICIÓN NUTRICIONAL DE LOS ALIMENTOS}

Se determinaron las concentraciones de proteína cruda (PC) y ceniza en los alimentos (AOAC 1996). La Fibra detergente neutro (FDN) fue determinada de acuerdo con Uden y col (2005), agregando $\alpha$ amilasa termoestable y utilizando un digestor de fibra TECNAL® TE-149.
ACTIVIDAD Y CONCENTRACIÓN DE PROTEÍNA EN LA ENZIMA

La actividad de la enzima amilolítica Hyrazime se determinó de acuerdo con Mendoza y col (1995) midiendo la cantidad de azúcares reductores totales después de la incubación de una solución de almidón de papa soluble con la $\alpha$-amilasa. La proteína que contiene el producto se midió con la técnica de Bradford (1976).

ENSAYO PRODUCTIVO, DIGESTIBILIDAD DE LA MATERIA

SECA, PATRÓN DE DISTRIBUCIÓN DEL CONSUMO

VOLUNTARIO DE ALIMENTO Y ACIDOSIS SUBCLÍNICA O CLÍNICA

Para estimar la ganancia diaria de peso (GDP) los corderos se pesaron individualmente antes de proporcionar el alimento, al inicio, a las cuatro semanas y al final del experimento. El experimento duró 56 días. La alimentación se ofreció una vez al día a las 08:00 horas y el consumo de alimento se calculó como la diferencia entre el alimento ofrecido y el rechazado en MS diariamente (Forbes 2007). La conversión alimentaria se definió como la cantidad de alimento en base seca requerido para obtener un $\mathrm{kg}$ de peso vivo.

Los días 54,55 y 56 del experimento se recogieron muestras individuales de alimento y heces procedentes de cinco corderos de cada tratamiento. Las cuales se analizaron para determinar la concentración de ceniza insoluble en ácido como marcador interno (Van Keulen y Young 1977) para estimar la digestibilidad de la MS.

Para establecer si la adición de enzima al concentrado en la dieta causó acidosis clínica o subclínica se analizó la distribución de la ingesta de alimento a lo largo del experimento para cada borrego mediante una prueba de Shapiro-Wilk. Se consideró que el animal sufría acidosis subclínica cuando su consumo se reducía más del $35 \%$ de su consumo promedio durante todo el experimento (Brown y col 2000). Para cada animal en el que se presentó dicha reducción se cuantificó el número de días que presentó la misma.

\section{ANÁLISIS ECONÓMICO}

Para este análisis se incluyó la estimación de los costos por la alimentación a lo largo del periodo y el ingreso por la venta de animales vivos. La diferencia entre estas dos variables se definió como utilidad neta. Los costos de los alimentos (US dólares) fueron para el concentrado comercial $\$ 360,00 /$ ton y heno de alfalfa $\$ 238,50 /$ ton. El costo de la enzima (Hyrazime) fue de $\$ 47,72 / \mathrm{kg}$ (US dólares).

\section{ANÁLISIS ESTADÍSTICO}

La ganancia diaria de peso, el consumo de alimento, la conversión alimentaria y la utilidad neta se analizaron 
mediante un modelo completamente al azar (Mendoza y col 2013), con nueve repeticiones para el tratamiento C y diez repeticiones para el tratamiento ENZ. La diferencia en el número de repeticiones entre los tratamientos fue causada por la muerte de un animal. El peso inicial de los animales se utilizó como covariable en el análisis estadístico de la ganancia de peso y el consumo de alimento. La digestibilidad de la MS fue analizada con el mismo modelo, pero utilizando cinco repeticiones por tratamiento (Lara y col 2013). La prueba de F se utilizó en todas las variables mencionadas anteriormente para determinar la significancia de las diferencias entre los tratamientos.

Los tres animales del tratamiento $\mathrm{Cy}$ los cinco animales del tratamiento ENZ que no presentaron una distribución normal del consumo a causa de los cambios en la ingesta del mismo y el número de días que el consumo de MS se mantuvo por debajo del $35 \%$ del valor promedio de cada animal fueron evaluados como variables cualitativas (Normal y No Normal; Consumo Promedio y Consumo Reducido) y comparados utilizando un análisis de contingencia. La prueba exacta de Fisher de dos colas se utilizó para establecer las diferencias entre tratamientos (Céspedes 2011). Todos los análisis fueron realizados utilizando el software JMP-7 (JMP 2007).

\section{RESULTADOS}

\section{COMPOSICIÓN NUTRICIONAL DE LOS ALIMENTOS}

La concentración de proteína cruda en el concentrado comercial y en el heno de alfalfa fue de 15,60 $\pm 0,31$ y $18,20 \pm 0,17 \%$. Mientras que la concentración de cenizas fue de 5,85 $\pm 0,22$ en el concentrado comercial y de 11,39 $\pm 0,02$ en el heno de alfalfa. La FDN fue de 28,61 $\pm 1,23$ y $36,06 \pm 0,46$ respectivamente y la composición estimada de la dieta fue de $16,38 \pm 0,17 \%$ PC, $7,51 \pm 0,16 \%$ cenizas y $30,85 \pm 0,61 \%$ de FDN.

\section{ACTIVIDAD ENZIMÁTICA}

La concentración de proteína en las muestras de enzima analizadas fue de $8,90 \% \pm 0,79$ y la actividad enzimática fue de 2,46 $\pm 0,075 \mathrm{~mol}$ de glucosa/mg de proteína minuto.

\section{ENSAYO PRODUCTIVO, DIGESTIBILIDAD DE LA MS, PATRÓN DE DISTRIBUCIÓN DEL CONSUMO VOLUNTARIO Y ACIDOSIS CLÍNICA Y SUBCLÍNICA}

El cuadro 1 presenta los pesos corporales iniciales y finales, el consumo promedio de alimento por día, la GDP, la conversión alimentaria y la digestibilidad aparente de la MS. No hubo diferencias significativas en las variables observadas. Sin embargo, el consumo de alimento tendió $(\mathrm{P}<0,07)$ a disminuir por efecto de la adición de enzima.

La prueba de Shapiro-Wilk mostró que el número de animales con una distribución anormal del consumo y una reducción mayor al 35\% fue de tres en el grupo control y seis en el grupo con enzima. Mientras que la prueba exacta de Fisher mostró que el número de animales con una reducción del consumo de alimento fue mayor en el tratamiento ENZ $\left(\mathrm{P}=1, \mathrm{R}^{2}=1\right)$ donde $\mathrm{p}$ es la probabilidad de que se repita el evento y $\mathrm{R}^{2}$ es el grado de asociación entre la enzima y la reducción del consumo de alimento; sin embargo, el número de días que los animales redujeron su consumo en promedio fue de seis para el grupo C y ocho para ENZ. De acuerdo con el análisis de contingencia, no hubo diferencias entre los tratamientos $(\mathrm{P}>0,05)$ en el número de días que los animales redujeron el consumo de MS.

\section{ANÁLISIS ECONÓMICO}

El beneficio económico para cada tratamiento se muestra en el cuadro 2. La utilidad por el uso de enzimas en la alimentación de rumiantes tiende a cero, a causa del aumento de los costos de alimentación por el costo de la adición de la enzima Hyrazime.

\section{DISCUSIÓN}

La actividad enzimática que Hyrazime mostró en este trabajo es similar o ligeramente superior a la publicada en otros estudios de $\alpha$ amilasas que provienen del Aspergillus

Cuadro 1. Efecto de la adición de $\alpha$ amilasa en el crecimiento y eficiencia de ovinos alimentados con dietas altas en grano. Effect of the addition of $\alpha$-amylase in the growth and efficiency of sheep fed high grain diets.

\begin{tabular}{|c|c|c|c|c|}
\hline & $\begin{array}{l}70 \% \text { concentrado } \\
\text { comercial }\end{array}$ & $\begin{array}{l}70 \% \text { concentrado } \\
\text { comercial + enzima }\end{array}$ & EEM & $\mathrm{P}$ \\
\hline Peso inicial, kg & 24,13 & 24,59 & 0,793 & 0,907 \\
\hline Peso final, $\mathrm{kg}$ & 37,40 & 37,60 & 0,858 & 0,669 \\
\hline Consumo de alimento, $\mathrm{kg}$ & 1,21 & 1,13 & 0,031 & 0,072 \\
\hline Ganancia diaria de peso, $\mathrm{kg}$ & 0,24 & 0,23 & 0,010 & 0,320 \\
\hline Conversión alimentaria & 5,00 & 5,01 & 0,199 & 0,950 \\
\hline Digestibilidad aparente de la MS, \% & 83,33 & 88,57 & 2,044 & 0,107 \\
\hline
\end{tabular}

EEM: error estándar de la media. 
Cuadro 2. Efecto de la adición de la $\alpha$ amilasa en la utilidad económica de corderos alimentados con dietas altas en grano. Effect of the addition of $\alpha$-amylase in the economic utility of lambs fed high grain diet.

\begin{tabular}{lcrrr}
\hline & $\begin{array}{c}70 \% \text { Concentrado } \\
\text { comercial }\end{array}$ & $\begin{array}{c}70 \% \text { Concentrado } \\
\text { comercial + enzima }\end{array}$ & EEM & P \\
\hline Costo de alimentación, dólares/periodo & 21,95 & 27,49 & 0,57 & 0,05 \\
Ingreso total por ventas, dólares & 29,25 & 27,57 & NS \\
Utilidad neta, dólares & 7,30 & 0,08 & 1,16 & 1,22 \\
\hline
\end{tabular}

EEM: error estándar de la media.

niger o del Bacillus licheniformis (Crosby 2008, Rojo y col 2005).

Los experimentos en ganado lechero utilizando $\alpha$ amilasa mostraron que la adición de la enzima, junto con el estado fisiológico de las vacas lecheras modifican el consumo de materia seca (DeFrain y col 2005). Esta interacción sugiere que en algunas ocasiones la enzima altera los patrones de fermentación ruminal que son necesarios para modificar el consumo de alimento (Allen 2000). En este experimento la enzima tendió $(P<0,07)$ a reducir el consumo de materia seca (figura 1A), lo que se asoció a la tendencia a aumentar la digestibilidad de la MS. La tendencia a modificar el consumo de alimento se podría explicar a partir de alteraciones en el patrón de alimentación (figura 1B), el mayor número de animales sin una distribución normal del consumo. Fulton y col
(1974) reportaron cambios en el patrón de consumo de alimento cuando se aumentaba la tasa de digestión del almidón en dietas altas en granos y lo asociaron con una reducción del $\mathrm{pH}$ ruminal que hace que el animal consuma una menor cantidad de alimento en un tiempo más largo. Trabajos evaluando los efectos in vitro de otras $\alpha$ amilasas muestran un aumento en la producción de AGV en forma paralela al aumento de la concentración de enzima (Klingerman y col 2009), lo que sugiere que la adición de la enzima aumenta la velocidad de digestión del almidón dietario, lo que conlleva a una reducción del pH y a una inhibición del consumo de alimento. Otros estudios realizados con novillos en corrales de engorda incluyendo $\alpha$ amilasa en la dieta, reportaron una tendencia a modificar el consumo de materia seca; sin embargo, no hubo cambios en el comportamiento productivo de los

Figura 1. Patrón de distribución del consumo voluntario en corderos alimentados con dietas altas en grano con y sin amilasa.

Distribution pattern of voluntary intake in lambs fed high grain diets with and without amylase.

A)

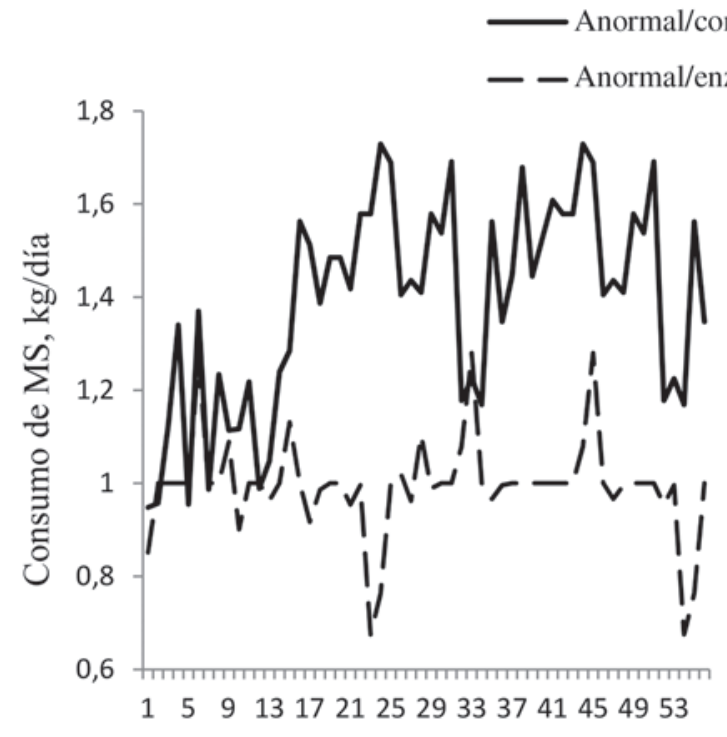

Días del experimento

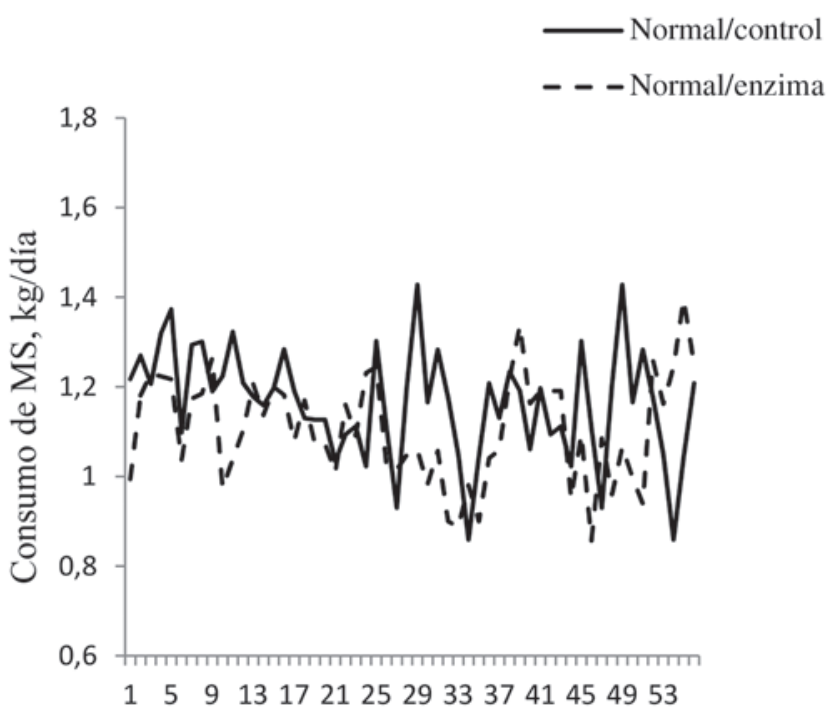

Días del experimento

A) Ejemplo de dos animales con ausencia de distribución normal (Shapiro-Wilks $\mathrm{p}<0.05$ ) de consumo voluntario.

B) Ejemplo de dos animales con una distribución normal (Shapiro-Wilks p > 0.1) de consumo voluntario.

A) Example of two animals without normal distribution (Shapiro-Wilks $\mathrm{p}<0.05$ ) of feed intake.

B) Example of two animals with normal distribution (Shapiro-Wilks $\mathrm{p}>0.1$ ) of feed intake. 
novillos (Tricarico y col 2007). Es importante destacar que estos autores encontraron una interacción entre la fuente de FDN y la enzima, de tal manera que cuando la fuente de fibra provenía de casquillo de algodón los resultados fueron mejores que cuando la fuente de fibra era alfalfa. Esto puede indicar que las enzimas generan cierto grado de acidosis que puede ser compensado con el aumento de la rumia. Lee-Rangel y col (2006) evaluaron el efecto del tratamiento de sorgo con la adición de la enzima en la alimentación de corderos y encontraron una tendencia $(\mathrm{P}<0,1)$ de la enzima a incrementar la digestibilidad de la MS y la MO. También sus datos mostraron que el consumo de materia seca fue $90 \mathrm{~g}$ menor en promedio con la enzima; sin embargo, no encontraron diferencias significativas entre tratamientos, lo que sugiere una gran variabilidad en el consumo de los animales. Estos mismos autores evaluaron la adición de un amortiguador con la finalidad de evitar la presentación de cuadros de acidosis al administrar la enzima; sus resultados mostraron nuevamente que no existían diferencias significativas $(\mathrm{P}>0,05)$ entre los tratamientos ni en el consumo ni en la ganancia de peso, pero el $\mathrm{pH}$ ruminal reportado para los tratamientos que contenían enzima con y sin amortiguador fueron menores en 0,2 y 0,3 con relación al testigo (Lee-Rangel y col 2010). En contraste, Crosby y col (2006) encontraron una reducción significativa ( $P$ $<0,05$ ) mayor al $9 \%$ en el consumo de alimento en los corderos que se alimentan con una dieta alta en grano cuando se les adicionó un nivel bajo de amilasa.

La ausencia de la distribución normal en el consumo de alimento fue reportada en vacas lecheras por Tolkamp y col (2000), quienes informaron una distribución aproximadamente normal pero ligeramente truncada del consumo voluntario. Esta alteración en el patrón de alimentación fue asociada a la tasa de consumo, el número de visitas al comedero y la hora a la que se ofreció el alimento. Forbes (2003) reportó una variación diaria en la ingesta de alimento; la cual fue explicada basándose en que el animal trata de minimizar la sensación de molestia ocasionada por estímulos asociados a la interacción entre su estado fisiológico (crecimiento, gestación, lactancia) y la dieta que consume (distensión ruminal, cambios en el pH, falta o exceso de nutrientes).

En este experimento, el tratamiento con enzima tuvo cinco animales sin una distribución normal de consumo de alimento, lo que se puede explicar porque la amilasa tendió $(\mathrm{P}<0,07)$ a reducir el consumo de alimento y puede estar asociada a la tendencia a aumentar la digestibilidad aparente de la materia seca $(\mathrm{P}<0,1)$. Este efecto había sido reportado por Rojo y col (2005) en los corderos alimentados con dietas altas en granos. Además, el aumento de la digestibilidad de la MS estuvo asociado con un cambio en la producción de $\mathrm{AGV}$ que reducen el $\mathrm{pH}$ y disminuyen el confort en el animal (Bevans y col 2005).

Se concluye que la inclusión de $\alpha$ amilasa en la dieta aumentó el número de animales con un patrón de consumo voluntario errático y el número de días que cada animal tuvo con un consumo de MS inferior al 35\% de su promedio, lo que sugiere una mayor incidencia de acidosis subclínica. Tampoco mejoró el comportamiento productivo y redujo la utilidad neta de los corderos en el periodo de finalización.

\section{REFERENCIAS}

Allen MS. 2000. Effects of diet on short-term regulation of feed intake by lactating dairy cattle. J Dairy Sci $83,1598-1624$.

AOAC, Association of Official Analytical Chemical. 1996. Official Methods of Analysis. 16 ${ }^{\text {th }} \mathrm{ed}$. AOAC, Washington, DC USA.

Bevans DW, KA Beauchemin, KS Schwartzkopf-Genswein, JJ McKinnon, TA McAllister. 2005. Effect of rapid or gradual grain adaptation on subacute acidosis and feed intake by feedlot cattle. J Anim Sci 83, 1116-1132.

Blanch M, S Calsamiglia, N DiLorenzo, A DiCostanzo, S Muetzel, RJ Wallace. 2009. Physiological changes in rumen fermentation during acidosis induction and its control using a multivalent polyclonal antibody preparation in heifers. J Anim Sci 87, 1722-1730.

Bradford MM. 1976. A rapid and sensitive method for the quantitation of microgram quantities of protein utilizing the principle of proteindye binding. Anal Biochem 72, 248-254.

Brown MS, CR Krehbiel, ML Galyean, MD Remmenga, JP Peters, B Hibbard, J Robinson, WM Moseley. 2000. Evaluation of models of acute and subacute acidosis on dry matter intake, ruminal fermentation, blood chemistry, and endocrine profiles of beef steers. J Anim Sci 78, 3155-3168.

Buendía RG, MGD Mendoza, GR Barcena, CME Ortega, HJ Solís, BA Lara. 2003. Efecto de la glucoamilasa de Aspergillus niger en la digestibilidad in vitro de maíz y sorgo y en la productividad de borregos. Agrociencia 37, 317-322.

Céspedes HJA. 2011. Presentación de alteraciones metabólico-nutricionales en rebaños lecheros pastoriles de cinco macrozonas en el sur de chile. Tesis de Magíster, Facultad de Ciencias Agrarias, Universidad Austral de Chile, Valdivia, Chile.

Colombatto D, FL Moulda, MK Bhat, E Owens. 2003. Use of fibrolytic enzymes to improve the nutritive value of ruminant diets. A biochemical and in vitro rumen degradation assessment. Anim Feed Sci and Tech 107, 201-209.

Crosby MM, GD Mendoza, LM Melgoza, R Barcena, FX Plata, E Aranda. 2006. Effects of Bacillus licheniformis amylase on starch digestibility and sheep performance. J Appl Anim Res 30,133-136.

Crosby MM. 2008. Evaluación de amilasas exógenas administradas en comprimidos matriciales inertes en el metabolismo ruminal. Tesis Dr. en Ciencias Biológicas, Universidad Autónoma Metropolitana, DF, México.

DeFrain JM, AR Hippen, KF Kalscheur, JM Tricarico. 2005. Effects of dietary $\alpha$-amylase on metabolism and performance of transition dairy cows. J Dairy Sc 88, 4405-4413.

DiLorenzo N, DR Smith, MJ Quinn, ML May, CH Ponce, W Steinberg, MA Engstrom, ML Galyean. 2011. Effects of grain processing and supplementation with exogenous amylase on nutrient digestibility in feedlot diets. Livest Sci 137, 178-184.

Forbes JM. 2003. The multifactorial nature of food intake control. J Anim Sci 81, E139-E144.

Forbes JM. 2007. Introduction. Methods of measuring food intake. In: Forbes JM (ed). Voluntary food intake and diet selection in farm animals. $2^{\text {nd }}$ ed. Biddles Ltd, King's Lynn, London, UK. Pp 1-40.

Fulton WR, TJ Klopfenstein, RA Britton. 1979. Adaptation to high concentrate diets by beef cattle. I. Adaptation to corn and wheat diets. J Anim Sci 49, 775-784.

Gaafar HMA, EM Abdel-Raouf, KFA El-Reidy. 2010. Effect of fibrolytic enzyme supplementation and fiber content of total mixed ration on productive performance of lactating buffaloes. Slovak J Anim Sci $43,147-153$. 
Gómez-Vázquez A, GD Mendoza, E Aranda, J Pérez, A Hernández, JM Pinos-Rodríguez. 2011. Influence of fibrolytic enzymes on growth performance and digestion in steers grazing stargrass and supplemented with fermented sugarcane. J Appl Anim Res 39, 77-79.

Huntington GB. 1997. Starch utilization by ruminants: From basics to the bunk. J Anim Sci 75, 852-867.

JMP release 7. 2007. Statistics and graphics guide. SAS Institute Inc., Cary, NC, USA.

Klingerman CM, W Hu, EE McDonell, MC DerBedrosian, L Kung Jr. 2009. An evaluation of exogenous enzymes with amylolytic activity for dairy cows. J Dairy Sci 92,1050-1059.

Lara BA, MGD Mendoza, GPA Hernández, GJA Martínez, PFX Plata. 2013. Evaluation of high doses of exogenous fibrolytic enzymes in lambs fed an oat straw based ration. Anim Nutr Feed Techn 13, 355-368.

Lee-Rangel HA, GD Mendoza, JM Pinos-Rodríguez, R Bárcena, F Plata, R Ricalde. 2006. Effect of an exogenous glucoamylase during different periods of time on performance of lambs fed sorghum based diets. J Appl Anim Res 29, 141-144.

Lee-Rangel HA, JM Pinos-Rodríguez, GD Mendoza, SS González, MA Montes, AS Trejo, Y Jasso-Pineda. 2010. Effect of a ruminal buffer and exogenous amylolytic enzymes on growth and digestion in lambs fed high concentrate diets. J Appl Anim Res 37, 117-120.

Mendoza MGD, RA Britton, RA Stock.1995. Effect of protozoa and urea level on in vitro starch disappearance and amylolytic activity of ruminal microorganisms. Anim Feed Sci Technol 54, 315-325.

Mendoza GD, N Mota, FX Plata, JA Martínez, PA Hernández. 2013. Effects of exogenous glucoamylase from aspergillus niger and grain level on performance of lambs. Anim Nutr Feed Techn 13, 391-398.

Mohamed DEDA, BE Borhami, KA El-Shazly, SMA Sallam. 2013. Effect of dietary supplementation with fibrolytic enzymes on the productive performance of early lactating dairy cows. J Agric Sci 5, 146-155.

Mota N, GD Mendoza, FX Plata, JA Martínez, H Lee, R Rojo, MM Crosby. 2011. Effect of exogenous glucoamylase enzymes and reduction of grain level on lamb performance. J Appl Anim Res 39, 129-131.
Owens FN, DS Secrist, WJ Hill, DR Gill. 1997. The effect of grain source and grain processing on performance of feedlot cattle: A review. J Anim Sci 75, 868-879.

Pinos JM, MS González, MGD Mendoza, JC García, L Miranda, G De la Cruz, V De Lerma. 2005. Efecto de enzimas fibrolíticas exógenas en la degradación in vitro de ingredientes alimenticios y en la producción de leche de vacas Holstein. Interciencia 30, 752-757.

Rode LM, TA McAllister, KA Beauchemin, DP Morgavi, VL Nsereko, WZ Yang, AD Iwaasa, Y Wang. 2002. Enzymes as direct-feed additives for ruminants. In: Renaville R, Burny A (eds). Biotechnology in animal husbandry. Vol. 5. Kluwer Academic Publishers, New York, USA, Pp 331-332.

Rojo R, GD Mendoza, SS González, L Landois, R Bárcena, MM Crosby. 2005. Effects of exogenous amylases from Bacillus licheniformis and Aspergillus niger on ruminal starch digestion and lamb performance. Anim Feed Sci and Tech 123-124, 655-665.

Tolkamp BJ, DPN Schweitzer, I Kyriazakis. 2000. The biologically relevant unit for the analysis of short-term feeding behavior of dairy cows. J Dairy Sci 83, 2057-2068.

Tricarico JM, MD Abney, ML Galyean, JD Rivera, KC Hanson, KR McLeod, DL Harmon. 2007. Effects of a dietary Aspergillus oryzae extract containing $\alpha$-amylase activity on performance and carcass characteristics of finishing beef cattle. J Anim Sci 85, 802-811.

Tricarico JM, JD Johnston, KA Dawson. 2008. Dietary supplementation of ruminant diets with an Aspergillus oryzae $\alpha$ amylase. Anim Feed Sci and Tech 145, 136-150.

Udén P, PH Robinson, J Wiseman. 2005. Use of detergent system terminology and criteria for submission of manuscripts on new, or revised, analytical methods as well as descriptive information on feed analysis and / or variability. Anim Feed Sci Tech 118, 181-186.

Van Keulen J, BA Young. 1977. Evaluation of acid-insoluble ash as a natural marker in ruminant digestibility studies. J Anim Sci 44, 282-287. 